\title{
Diffusivity scaling on shear flow
}

\author{
Zhong-Tian Wang $^{1,2}$, Zhi-Xiong He ${ }^{1}$, Jia-Qi Dong ${ }^{1}$, Zhan-Hui Wang ${ }^{1}$, Shao-Yong Chen ${ }^{2}$, \\ Chang-Jian Tang ${ }^{2}$
}

${ }^{1}$ Southwestern Institute of Physics, Chengdu, Sichuan, 610041, China

${ }^{2}$ College of Physics Science and Technology, Sichuan University, Chengdu, Sichuan, 610065, China

\section{Email address:}

wangzt@swip.ac.cn (Zhong-Tian Wang)

\section{To cite this article:}

Zhong-Tian Wang, Zhi-Xiong He, Jia-Qi Dong, Zhan-Hui Wang, Shao-Yong Chen, Chang-Jian Tang. Diffusivity Scaling on Shear Flow. American Journal of Modern Physics. Vol. 3, No. 5, 2014, pp. 202-206. doi: 10.11648/j.ajmp.20140305.12

\begin{abstract}
Diffusivity scaling on shear flow is investigated. Radial electrical field is the drive of the flow. The turning points of the trapped particle are not on the drift surface, but modified by the radial electrical field. For the first time, an analytical expression of the banana width in presence of shear flow is accurately derived. The particle diffusivity given by Rosenbluth is reproduced but with the shear flow modification.
\end{abstract}

Keywords: Tokamak Plasma, Diffusivity Scaling, Shear Flow, Trapped Particle, Guiding-Center

\section{Introduction}

It is generally claimed that the shear flow plays an important role in the onset of the transition from the $\mathrm{L}$ mode to H-mode. Experimental evidence ${ }^{1}$ also showed that the plasma rotates rapidly in the improved confinement regime, implying that the radial electric field is generated. Neoclassical ion transport in rotating axisymmetric plasma has been systematically investigated by Hinton ${ }^{2}$. However, the energy loss sometimes in the experiment is smaller than the standard neoclassical prediction. The improvement is attributed to the squeezing factor ${ }^{3}$ treated as the shear flow in this paper. There are renewed interests in the neoclassical fluxes $^{4-6}$ inside the thermal barriers. The diffusivity scaling on the shear flow presented by Catto $^{6}$ and Shaing ${ }^{5}$ are different. There are intense arguments ${ }^{6}$ on this topic. It is important because it is related to the role of the shear flow in the thermal barrier. Physicists should give a definite answer. The discrepancy between Catto ${ }^{6}$ and Shaing ${ }^{5}$ comes from the trapped particle dynamics which is emphasized in this paper. Accurate banana width, bounce frequency, and the turning point position in the presence of the shear flow are presented. Drift kinetic equation is solved with a particle-conserved Krook collision operator. Rosenbluth's result $^{7}$ is reproduced but with the shear flow modification. The fraction of the trapped particles estimated here is the same as Shaing's ${ }^{5}$, however, diffusivity scaling on shear flow is different.

In section 2, a set of canonical guiding-center variables is derived by area-conserved transformation. Dynamics for the trapped particles is given in section 3. The drift kinetic equation is solved and the diffusivity scaling is derived in section 4. Summary is presented in the last section.

\section{Canonical Guiding-Center Variables}

For a tokamak configuration, the Hamiltonian of a charged particle can be expressed as:

$$
\begin{aligned}
& H=\frac{1}{2 M}\left[\left(P_{R}-e A_{R}\right)^{2}+\left(P_{Z}-e A_{Z}\right)^{2}\right. \\
& \left.+\left(P_{\phi}-e R A_{\phi}\right)^{2} / R^{2}\right]+e \Phi
\end{aligned}
$$

where $A_{R}, A_{Z}$, and $A_{\phi}$ are the vector potential components of the magnetic field, $\Phi$ the electrical potential assumed to be a function of the poloidal magnetic flux $\Psi, M$ the mass of the charged particle set equal to unity for simplicity, and $e$ the charge. $P_{R}, P_{\phi}$ and $P_{Z}$ are the canonical momentum in the cylindrical coordinates $R, \phi$, and $Z$, respectively, which are as following:

$$
\begin{gathered}
P_{R}=v_{R}+e A_{R}, \\
P_{\phi}=R v_{\phi}+e \mathrm{R} A_{\phi}, \\
P_{Z}=v_{Z}+e A_{Z} .
\end{gathered}
$$


The magnetic field in tokamaks can be expressed as:

$$
B=\nabla \phi \times \nabla \Psi+I \nabla \phi
$$

where $I$ is related to the poloidal current. The vector potential components are

$$
A_{R}=0, A_{Z}=-I \ln \frac{R}{R_{0}}, A_{\phi}=-\frac{\Psi}{R} .
$$

As stated by Littlejohn, there has been a gradual evolution over the years away from the averaging approach and towards the transformation approach ${ }^{8}$. We introduce a generating function ${ }^{9}$ to change the cylindrical variables to guiding-center variables:

$$
F_{1}=-\frac{\Omega_{0} R_{0}^{2}}{2} \exp \left(\frac{X}{\Omega_{0} R_{0}}\right)\left(\ln \frac{R}{R_{0}}-\frac{X}{\Omega_{0} R_{0}}\right)^{2} \operatorname{tg} \alpha-Z X
$$

where

$$
X=\Omega_{0} R_{0} \ln \frac{R_{C}}{R_{0}}
$$

$\Omega_{0}$ is the toroidal gyrofrequency at the magnetic axis, $\rho$ the Larmor radius, $\alpha$ the gyrophase, and the subscripts o and $c$ refer to the values at the magnetic axis and the guiding center, respectively. $X$ and $\alpha$ are the new coordinates conjugate to the new momenta:

$$
\begin{gathered}
P_{X}=Z+\rho \sin \alpha+\frac{\rho^{2}}{4 R_{C}} \sin 2 \alpha \\
P_{\alpha}=\frac{1}{2} \Omega_{C} \rho^{2}
\end{gathered}
$$

where $P_{X}$ is the guiding center of the $Z$ coordinate, $Z_{c}$. The momentum is often transformed into the coordinate during area-conserved canonical transformation ${ }^{9}$.

The Hamiltonian is rewritten as:

$$
H=\Omega_{C} P_{\alpha}\left[\left(\frac{R_{C}}{R}\right)^{2} \sin ^{2} \alpha+\cos ^{2} \alpha\right]+\frac{1}{2 R^{2}}\left[P_{\varphi}+e \Psi\right]^{2}+e \Phi .
$$

The canonical transformation makes the Hamiltonian exact in the new coordinates. The canonical guiding-center variables, $P_{\alpha}, P_{\phi}, P_{X}, \alpha, \phi, X$, are derived and satisfy the Hamiltonian equations:

$$
\begin{gathered}
\dot{P}_{i}=-\frac{\partial H}{\partial q_{i}} \\
\dot{q}_{i}=\frac{\partial H}{\partial P_{i}},
\end{gathered}
$$

where the $P$ and $q$ are known as the generalized momenta and coordinates. The Jacobian is unity for the area-conserved transformation ${ }^{9}$, that is,

$$
J=d P_{\alpha} d P_{X} d P_{\phi} d \alpha d X d \phi=1
$$

For the tokamaks, the ordering is

$$
\delta \sim \rho / r \sim r / R \sim B_{\theta} / B_{\phi}
$$

where $r$ and $R$ are the minor and major radii respectively and $R=R_{0}+r \cos \theta$. To the first order, the gyro-averaged Hamiltonian in Eq. (11) is approximately expressed as:

$$
H=P_{\alpha} \Omega_{c}+\frac{1}{2 R^{2}}\left(P_{\xi}+e \Psi_{c}\right)^{2}+e \Phi_{c}
$$

We have a set of equations of motion in the guiding-center system:

$$
v_{\Psi}=\frac{B_{R}}{B_{p}} v_{\mathrm{d}}
$$

$$
v_{p}=\frac{B_{p}}{B_{\varphi}}\left(v_{\phi}-\frac{R v_{E}}{R_{0}}\right)+\frac{B_{z}}{B_{p}} v_{\mathrm{d}}
$$

where $\quad v_{\varphi}=\left(P_{\phi}+e \Psi_{c}\right) / R \quad, \quad v_{E}=-R_{0} \frac{\partial \Phi}{\partial \Psi} \quad, \quad$ and $v_{\mathrm{d}}=\frac{1}{\Omega_{0} R_{0}}\left(\Omega_{c} \mu+v_{\phi}^{2}\right), v_{\Psi}$ is in the radial direction whereas $v_{\mathrm{p}}$ is in the poloidal direction. Equations (17) and (18) are the generalized versions of the equations of motion obtained by Balescu $^{10}$.

\section{Dynamics for the Trapped Particles}

For the trapped particles, the toroidal velocity is smaller than the perpendicular velocity,

$$
v_{\phi} / v_{\perp}=v_{\phi} /\left(2 \Omega_{c} P_{\alpha}\right)^{\frac{1}{2}} \sim \delta^{\frac{1}{2}}
$$

In the rotation frame, we can construct a Hamiltonian in the developed canonical variables, $P_{\alpha}, P_{\phi}, P_{X}, \alpha, \phi, X$,

$$
\begin{aligned}
& H=\Omega_{0} P_{\alpha} e^{-\frac{X}{\Omega_{0} R_{0}}}+\frac{1}{2 R_{o}^{2} S} e^{-\frac{2 X}{\Omega_{0} R_{0}}}\left[P_{\varphi}+e \Psi-R_{0} v_{E} e^{\frac{2 X}{\Omega_{0} R_{0}}}\right]^{2}, \\
& =\frac{1}{2} u_{\perp}^{2}+\frac{1}{2} u_{\phi}^{2}
\end{aligned}
$$

where $u_{\perp}=v_{\perp}, u_{\phi}=\frac{P_{\phi}+e \Psi-R v_{E}}{S^{\frac{1}{2}} R} \approx \frac{v_{\phi}}{S^{\frac{1}{2}}}, S=1+\frac{\rho_{p}^{2} e \Phi^{\prime \prime}(r)}{2 T_{i}}$, $\rho_{p}$ is the poloidal Larmor radius, $v_{E} / v_{t}$ is the order of $\delta$, $v_{t}=\sqrt{2 T / M}$ is the particle thermal velocity, $\mathrm{S}$ is the squeezing factor, the shear flow. The different forms of the Hamiltonian describe the same motion. Eqs.(17) and (18) can be reproduced from Eq.(20).

With the small inverse aspect ratio approximation, we have 


$$
\begin{gathered}
u_{\phi}=u_{\phi 0} \sqrt{1-k^{2} \sin ^{2} \frac{\theta}{2}}, \\
u_{\phi 0}=\left[2 H-2 \Omega_{0} P_{\alpha}\left(1-\frac{r_{0}}{R_{0}}\right)\right]^{\frac{1}{2}}, \\
k=\left(\frac{2 \varepsilon u_{\perp}^{2}}{u_{\phi 0}^{2}}\right)^{\frac{1}{2}} .
\end{gathered}
$$

The $r_{0}$ should be banana center position. The turning points $\theta_{t}$ of the banana orbit are decided by the following formula,

$$
k^{2} \sin ^{2} \frac{\theta_{t}}{2}=1
$$

Once $\theta_{t}=\pi$ we have $k^{2}=1$ and $u_{\phi \max }=\left(2 \varepsilon u_{\perp}^{2}\right)^{\frac{1}{2}}$ for the trapped particles.

We set $\Psi_{0}$ as the banana center surface where turning points are evaluated and then we expand $\Psi$ and $v_{E}$ near $\Psi_{0}$. The banana width and the position of banana center surface are obtained after the expansion,

$$
u_{\phi}=\frac{P_{\phi}+e \Psi-R v_{E}}{S^{\frac{1}{2}} R}=\frac{P_{\phi}+e \Psi_{0}-R v_{E 0}+S R \Omega_{p} \Delta}{S^{\frac{1}{2}} R},
$$

where $\Delta$ is the banana width,

$$
\begin{gathered}
\Delta=\frac{u_{\phi}}{S^{\frac{1}{2}} \Omega_{p}}=\frac{u_{\phi 0}}{S^{\frac{1}{2}} \Omega_{p}} \sqrt{1-k^{2} \sin ^{2} \frac{\theta}{2}}, \\
\Psi_{0}=\Psi_{*}+R v_{E 0} / e,
\end{gathered}
$$

where $\Psi_{*}=-P_{\phi} / e$ is drift surface and $v_{E 0}$ is the value of $v_{E}$ on the banana center surface. We can see that the turning points of the trapped particle are not on the drift surface but shifted to banana center surface due to the radial electrical field.

We form an invariant ${ }^{9}$ variable,

$$
\Pi=\frac{1}{2 \pi} \oint P_{X} d X=\frac{\Omega_{0}}{2 \pi} \oint Z_{c} \frac{R_{0}}{R_{c}} d R_{c} \approx \frac{\Omega_{0}}{2 \pi} \oint r_{0} \Delta d \theta,
$$

which is actually the flux enclosed by banana orbit. Now we introduce a new angle which satisfies the equation,

$$
\sin ^{2} \beta=k^{2} \sin ^{2} \frac{\theta}{2}
$$

then,

$$
\begin{aligned}
& \Pi \approx \frac{\Omega_{0} r_{0} u_{\phi 0}}{\pi S^{\frac{1}{2}} \Omega_{p}} \int_{-\theta_{t}}^{\theta_{t}} \sqrt{1-k^{2} \sin ^{2} \frac{\theta}{2}} d \theta=\frac{4 q R_{0} u_{\phi 0} k_{1}}{\pi S^{\frac{1}{2}}} \int_{0}^{\frac{\pi}{2}} \frac{\left(1-\sin ^{2} \beta\right) d \beta}{\sqrt{1-k_{1}^{2} \sin ^{2} \beta}}, \\
& =\frac{8 q R_{0}\left(e \Omega_{0} P_{\alpha}\right)^{\frac{1}{2}}}{\pi S^{\frac{1}{2}}}\left[E\left(k_{1}\right)-\left(1-k_{1}^{2}\right) K\left(k_{1}\right)\right]
\end{aligned}
$$

where $k_{1}^{2}=k^{-2}, K$ and $E$ are complete elliptic functions. The bounce frequency of the trapped particle is

$$
\omega_{b}=\frac{\partial H}{\partial \Pi}=\frac{\left(2 \varepsilon S v_{\perp}^{2}\right)^{\frac{1}{2}}}{2 q R_{0}}\left(\frac{\pi}{2 K\left(k_{1}\right)}\right)
$$

Eqs. (21-31) give, for the first time, a so clear picture of the trapped particle motion dependent on the shear flow. It is real motion no other choice.

For a Maxwellian distribution function, the fraction of the trapped particles can easily calculated via

$$
F=2 \sqrt{\pi} \int_{0}^{\infty} y d y \int_{-x_{\max }}^{x_{\max }} e^{-y^{2}-x^{2}} d x
$$

where $y=v_{\perp} / v_{t}, x=v_{\phi} / v_{t}$, and

$v_{\phi \max }=S^{\frac{1}{2}} u_{\phi \max }=S^{\frac{1}{2}}\left(2 \varepsilon v_{\perp}^{2}\right)^{\frac{1}{2}}$. For the trapped particles, $\mathrm{x}$ is small, thus it leads

$$
F=4 \sqrt{\pi} \int_{0}^{\infty} d y e^{-y^{2}} y^{2}(2 \varepsilon S)^{\frac{1}{2}}=(2 \varepsilon S)^{\frac{1}{2}},
$$

which agrees with Shaing's 5 and disagrees with Catto's 6 . Since the trapped particle pitch angle is limited, the effective collision frequency $v_{\text {eff }}$ here should be $v /\left(u_{\phi}^{2} / v^{2}\right)$. Thus, the diffusivity is $F \Delta^{2} v v^{2} / u_{\phi}^{2} \approx v \rho_{p}^{2}(2 \varepsilon / S)^{\frac{1}{2}}$ which is different with Shaing's 5 .

\section{Neoclassical Transport}

To illustrate the significance of the trapped-particle dynamics, the particle diffusivity is calculated. Instead of canonical gyrokinetic variables, $P_{\alpha}, P_{\phi}, P_{X}, \alpha, \phi, X$, we use extended phase space variables ${ }^{11}, P_{\alpha}, P_{\phi}, r, H, \alpha, \phi, \beta, t$, in the drift kinetic equation,

$$
\frac{\partial f}{\partial t}+\frac{d \beta}{d t} \frac{\partial f}{\partial \beta}+\frac{d r}{d t} \frac{\partial f}{\partial r}=C(f) .
$$

We define an averaged angle velocity,

$$
<\frac{d \beta}{d t}>=\frac{1}{T} \oint \frac{d \beta}{d t} d t=\frac{2 \pi}{T},
$$


where $T$ is the bounce period. We can see that the averaged angle velocity is the bounce frequency in Eq. (31). To illustrate flow shear effects on the neoclassical transport, we take

$$
f=f_{*}+g=f_{*}+g_{0}+g_{1 s} \sin \beta+g_{1 c} \cos \beta,
$$

where $f_{*}$ is equilibrium distribution function in a Maxwellian form with $\mathrm{H}$ at the place of energy and $P_{\phi}$ at the place of position,

$$
\begin{gathered}
f_{*}=F_{m}(r)\left[1-\frac{v_{\phi}}{\Omega p} \frac{\partial \ln n}{\partial r}\right], \\
\frac{\partial f_{*}}{\partial r}=0 .
\end{gathered}
$$

To catch the key points and avoid the complexity we only consider density gradient. Temperature gradient can change the expression of diffusivity, but can not change the scaling on the shear flow.

Eq. (34) is rewritten as

$$
\begin{gathered}
\frac{\partial \beta}{\partial t} \frac{\partial g}{\partial \beta}-\sin \theta v_{d} \frac{\partial g}{\partial r}=C\left(g-g_{m}\right)-\frac{\partial f}{\partial t}, \\
g_{m}=\frac{v_{\phi}}{\Omega_{p}} F_{m} \frac{\partial \ln n}{\partial r} .
\end{gathered}
$$

To make Eq. (39) tractable, we take

$$
\frac{d \beta}{d t} \approx<\frac{d \beta}{d t}>=\omega_{b}
$$

and

$$
C\left(g-g_{m}\right)=v_{e f f}\left(g-g_{m}\right) .
$$

Furthermore, it is assumed that $k_{1}$ and $\theta_{t}$ are small, which means deeply trapped particles dominate. In Eq. (36) $\mathrm{g}_{1 \mathrm{~s}}$ and $\mathrm{g}_{1 \mathrm{c}}$ are one order smaller than $\mathrm{g}_{0}$. From Eq. (29) and Eq. (39) we obtain $g_{0}=g_{m}, g_{1 s}=\frac{2 k_{1} v_{e f f} \frac{\partial g_{0}}{\partial r}}{\omega_{b}^{2}+v_{e f f}^{2}}, g_{1 c}=-\frac{\omega_{b}}{v_{e f f}}$.

With Eq. (37) and Eq. (38) the drift equation of Eq. (39) after the bounce average turns to be

$$
\frac{\partial f}{\partial t}-\frac{\partial}{\partial r} \frac{2 v_{e f f} k_{1}^{2} v_{d}^{2}}{\omega_{b}^{2}+v_{e f f}^{2}} \frac{\partial F_{m}}{\partial r}=0
$$

In the banana regime, we have

$$
\frac{v_{e f f}^{2}}{\omega_{b}^{2}} \approx \delta
$$

After integrating Eq. (43) over velocity space, we get the continuity equation

$$
\frac{\partial n}{\partial t}+\frac{\partial}{\partial r} \Gamma=0
$$

where $\Gamma=-D \frac{\partial n}{\partial r}$,

$$
D=2 \int_{0}^{\infty} y d y \int_{-x_{\max }}^{x_{\max }} d x \frac{2 v_{e f f} k_{1}^{2} v_{d}^{2}}{\sqrt{\pi} \omega_{b}^{2}} e^{-y^{2}-x^{2}}=0.75 v \sqrt{2 \varepsilon} \rho_{p}^{2} / S^{\frac{1}{2}},
$$

where $x=\frac{v_{\phi}}{v_{t}}, y=\frac{v_{\perp}}{v_{t}}, x_{\max }=\frac{\sqrt{2 \varepsilon S} v_{\perp}}{v_{t}}=\sqrt{2 \varepsilon S} y$. From Eq.(46) we can see that Rosenbluth's result ${ }^{9}$ is reproduced but with the shear flow modification.

\section{Summary}

The area-conserved transformation proposed by Lichtenberg and Lieberman9 is employed. A complete set of canonical guiding-center variables, $P_{\alpha}, P_{\phi}, P_{X}, \alpha, \phi, X$, are derived. The accurate relation between the particle motion and the shear flow for the trapped particles is also derived, including the banana width $\Delta=\frac{u_{\phi}}{S^{\frac{1}{2}} \Omega_{p}}=\frac{u_{\phi 0}}{S^{\frac{1}{2}} \Omega_{p}} \sqrt{1-k^{2} \sin ^{2} \frac{\theta}{2}}$, the position of banana center surface $\Psi_{0}=\Psi_{*}+R v_{E 0} / e$ and the bounce frequency $\omega_{b}=\frac{\partial H}{\partial \Pi}=\frac{\left(2 \varepsilon S v_{\perp}^{2}\right)^{\frac{1}{2}}}{2 q R_{0}}\left(\frac{\pi}{2 K\left(k_{1}\right)}\right)$. For a Maxwellian distribution function, the fraction of the trapped particles is calculated as $F=4 \sqrt{\pi} \int_{0}^{\infty} d y e^{-y^{2}} y^{2}(2 \varepsilon S)^{\frac{1}{2}}=(2 \varepsilon S)^{\frac{1}{2}}$ which agrees with Shaing's5 and disagrees with Catto's6. Since the trapped particle pitch angle is limited, the effective collision frequency $v_{\text {eff }}$ here should be $v /\left(u_{\phi}^{2} / v^{2}\right)$, therefore, the diffusivity is $F \Delta^{2} v v^{2} / u_{\phi}^{2} \approx v \rho_{p}^{2}(2 \varepsilon / S)^{\frac{1}{2}}$ which is different with Shaing's5. Drift kinetic equation is solved with particle-conserved Krook collision operator. Rosenbluth's result7 is reproduced but with shear flow modification in this paper.

\section{Acknowledgments}

It is appreciated that Dr. R. D. Hazeltine and Dr. P. Morrision checked and verified the generating function when author Z. T. Wang worked at IFS of the University of Texas.

This work was supported by Natural Science Fund No. 11261140327, No.11205053, No.11005035, No.11105046 and The National Magnetic Confinement Fusion Science Program (Grant No. 2013GB107002). 


\section{References}

[1] E.J.Doyle, R.J. Groebner, K.M.Burrell, P. Gohil, T. Lehcka, N. C. Luhmann Jr., H. Matsumoto, T. H. Osborne, W. A. Peebles, and R. Philipona, Modification in turbulence and edge electric field at the L-H transition in Dlll-D tokamak, Phys. Fluids B 3, 2300(1991).

[2] F.L.Hinton and S.K.Wong, Neoclassical ion transport in rotating axisymmetrical plasmas, Phys. Fluids 28, 3082(1985).

[3] R.D.Hazeltine, Self-consistent radial sheath, Phys.Fluids B1, 2031(1989).

[4] G. Kagan and P. J. Catto, Phys. Rev. Lett. 105, 045002(2010).

[5] K. C. Shaing and C. T. Hsu, Phys. Plasmas 19, 022502(2012).

[6] P. J. Catto, F. I. Parra, G. Kagan, J. B. Parker, I. Pusztai, and M. Landreman, Plasma Phys. Control. Fusion, 55, 045009(2013).

[7] M. N. Rosenbluth, R. D. Hazeltine, F. L. Hinton, Phys. Fluids, 15, 116(1972).

[8] R. G. Littlejohn, J. Plasma physics 29, 111(1983).

[9] A. J. Lichtenberg and Lieberman, Regular and Stochastic Motion, Applied Sciences 38, (Springer-Verlag New York Inc. 1983).

[10] R. Balescu, in Transport Processes in Plasma, (North-Holland, Amsterdam · Oxford · New York · Tokyo, 1988), Vol.2, P.393. 